\title{
Can sleep microstructure improve diagnosis of OSAS? Integrative information from CAP parameters
}

\author{
G. MILIOLI', M. BOSI'², A. GRASSI', S. RICCARDI', M.G. TERZANO', P. CORTELLI3,4, \\ V. POLETT|2,5, L. PARRINO'
}

${ }^{1}$ Sleep Disorders Center, Dept of Neurosciences, University of Parma, Italy; ${ }^{2}$ Pulmonary Operative Unit, Dept of Thoracic Diseases, G.B. Morgagni - L. Pierantoni Hospital, Forlì, Italy; ${ }^{3}$ IRCCS, Institute of Neurological Sciences of Bologna, Bologna, Italy; ${ }^{4}$ Department of Biomedical and NeuroMotor Sciences (DIBINEM), Alma Mater Studiorum, University of Bologna, Italy, ${ }^{5}$ Department of Respiratory Diseases \& Allergology, Aarhus University Hospital, Aarhus (DK)

\section{A B S T R A C T}

Purpose: The scoring of American Academy of Sleep Medicine (AASM) arousal is mandatory for the definition of respiratory event-related arousal (RERA). However there are other EEG activation phenomena, such as A phases of cyclic alternating pattern (CAP) which are associated with respiratory events in non rapid eye movements (NREM) sleep.

This study aims at quantifying the additional value of CAP for the definition of respiratory events and sleep alterations in OSAS. Methods: Analysis of polysomnographic recordings from nineteen OSAS patients was carried out. Scoring was focused on investigation of the cerebral response to flow limitation (FL) events. For this purpose we used both CAP rules and AASM arousal criteria.

Main Results: While no difference was demonstrated in the arousal index between mild and moderate-severe OSAS patients, CAP time showed a progressive enhancement from normal subjects $(152,5 \pm 20,76)$ to mild $(180,64 \pm 34,76)$ and moderatesevere $(282,27 \pm 58,02)$ OSAS patients $(p<0.0001)$.

In NREM sleep, only $41.1 \%$ of FL events met the criteria for the definition of RERA, while, $75.5 \%$ of FL events ended with a CAP A phase and most FL CAP (69.1\%) terminated with a CAP phase A3 subtype.

Conclusions: Our data indicate that the RERA scoring has a limited accuracy in the detection of FL events. In NREM sleep, $C A P$ rules provided more information than AASM arousal for the definition of respiratory events and sleep alterations in OSAS.

Key Words

Obstructive Sleep Apnea Syndrome $\bullet$ Cyclic Alternating Pattern $\bullet$ Arousal $\bullet$ Flow Limitation Events

\section{Introduction}

Obstructive Sleep Apnea Syndrome (OSAS) is a common disorder characterized by repetitive collapse of the upper airways during sleep, leading to episodes of complete (apnea) or partial (hypopnea) upper airway obstruction. These respiratory events often result in reductions in blood oxygen saturation and brief arousals from sleep. Clinically, OSAS is defined by the occurrence of daytime sleepiness, loud snoring, witnessed breathing interruptions, or awakenings due to gasping or choking in the presence of at least 5 obstructive respiratory events (apneas, hypopneas or respiratory effort related arousals) per hour of sleep. The Apnea Hypopnea Index (AHI) is the most common parameter applied for the severity classification of OSAS. However, arousal scoring in OSAS patients is also important to clarify the impact of this disease on sleep (Thomas 2003; Masa et al. 2013). Chandra et al. (Chandra et al. 2013) have demonstrated that patients with a high RERA (respiratory event related arousal) index, even in the setting of 
a low or normal AHI, may be exposed to elevated sympathetic tone during sleep.

The RERA definition is based on the American Academy of Sleep Medicine (AASM) criteria for arousals (Iber C et al. 2007), which however underestimate more subtle phasic activations of sleep (Thomas 2003; Jordan et al. 2011; Dingli et al. 2002), both in normal subjects and in pathologic conditions.

In OSAS patients, the multiple airflow interruptions followed by arousals show a 20- to 40-s cyclic pattern. The periodicity of the OSAS-related phenomena recalls the physiological component of non rapid eye movements (NREM) sleep, which appears independently of breathing alterations or other sleep pathologies and which is known as the cyclic alternating pattern (CAP) (Terzano et al. 1985). Functionally, CAP translates a condition of sustained arousal instability between levels of activation (phase A) and deactivation (phase B) in NREM sleep (Parrino et al. 2012).

In the CAP framework, the cerebral response is a complex phenomenon in which the arousal definition is more extensive compared to the AASM criteria(Iber C et al. 2007), as it incorporates not only the low-voltage high-frequency EEG bands such as alpha and beta (AASM arousal or subtypes A2 and A3 of CAP A phases), but also the highvoltage lower frequency activities such as theta and delta waves (A1 CAP A phases) (Figure. 1).

It is known that, compared to normal subjects, OSAS patients show enhanced amounts of CAP with the great majority of apneas (AP) and hypopneas $(\mathrm{H})$ ending with CAP A phases, especially subtypes A2 and A3 subtypes (Terzano et al. 1996). In contrast, there are no available data on the relation between flow limitation (FL) events and CAP parameters and the impact of FL events on sleep is still unclear. The present study aims at assessing in OSAS patients the impact of FL events on sleep and whether CAP represents an additional value, compared to the currently applied AASM arousal criteria, for a more accurate detection of subtle respiratory events and alterations of sleep in OSAS patients.

\section{Materials}

Polysomnography (PSG) recordings of nineteen consecutive OSAS patients (males, mean age $54 \pm 10$ ), were carried out at the Forlì Hospital Pulmonary Operative Unit sleep laboratory between March 2012 and August 2012. The institutional review board approved the analysis of PSG data.

The full-night attended PSG was performed in each patient with Embla ${ }^{\circledR}$ Titanium (Embla Systems). Sleep was recorded from F4-M1, C4-M1, O2-M1 derivations integrated by bipolar montages (Fp2-F4, F4-C4, C4-P4, $\mathrm{P} 4-\mathrm{O} 2$ ) used to optimize the scoring of CAP (Terzano et al. 2002). PSG measures included also electrooculographic channels, submental electromyogram, activity of the right and left anterior tibialis muscles, oronasal thermal sensor, nasal air pressure transducer, thoracic and abdominal effort by respiratory inductive plethysmograph, electrocardiogram, body position, oxygen saturation and pulse wave amplitude.

\section{Methods}

The PSG interpretation, carried out by two experienced sleep investigators (1 pulmonologist, 1 neurologist) was performed in three different steps:

1) scoring of respiratory events (AP, H, FL) based on visual analysis of respiratory traces;

2) scoring of EEG activity, applying both the AASM criteria and the CAP rules;

3) temporal relation between arousal responses and FL events.

Only the FL events that met the agreement of both scorers were included in the data analysis.

OSAS patients were divided in two severity groups. According to a consensus opinion of the American Academy of Sleep Medicine Task Force (Anon 1999), the mild group was composed of patients with an $5 / \mathrm{h}<\mathrm{AHI}<15 / \mathrm{h}$, while an $\mathrm{AHI}>15 / \mathrm{h}$ characterized the moderate-severe group.

\section{Scoring of Respiratory Events}

The respiratory events were defined according to the AASM rules (Iber C et al. 2007). Obstructive apnea $(\mathrm{OA})$ was defined as a reduction in the oral thermistor signal to less than $10 \%$ of baseline, lasting at least 10 seconds with continued or increased respiratory effort. Central apnea (CA) was identified when there was absence of respiratory effort. Mixed apnea (MA) was scored when a respiratory effort was absent in the initial portion of the event, followed by resumption of inspiratory effort in the 


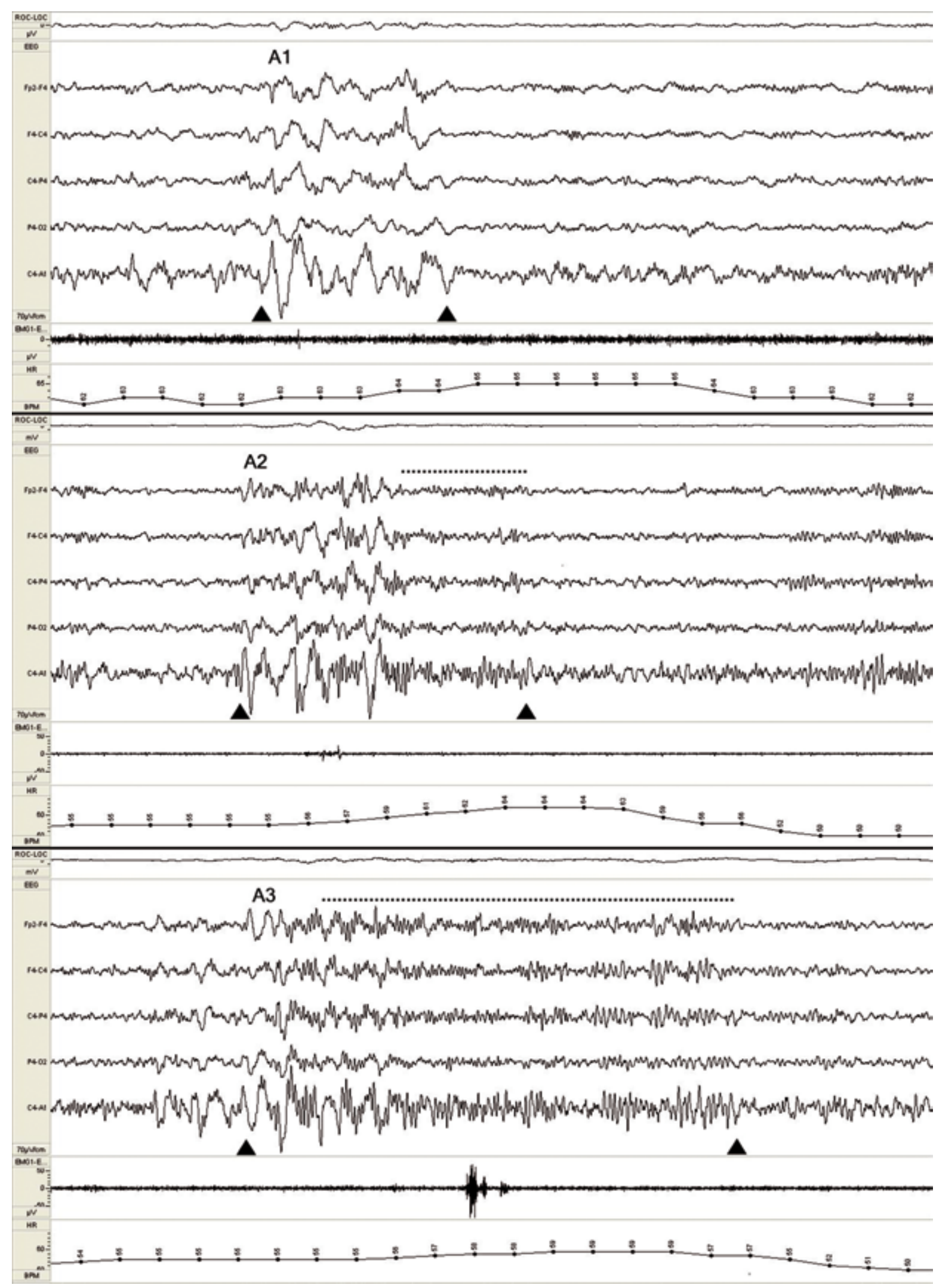

Fig. 1. - Phase A subtype. The dotted spots indicate the fast low-amplitude portion of the phase A. Abbreviations. ROC-LOC: electrooculographic channels; EEG: electroencephalographic traces; EMG: submental electromyogram; HR:heart rate. 


\section{CAP Scoring}

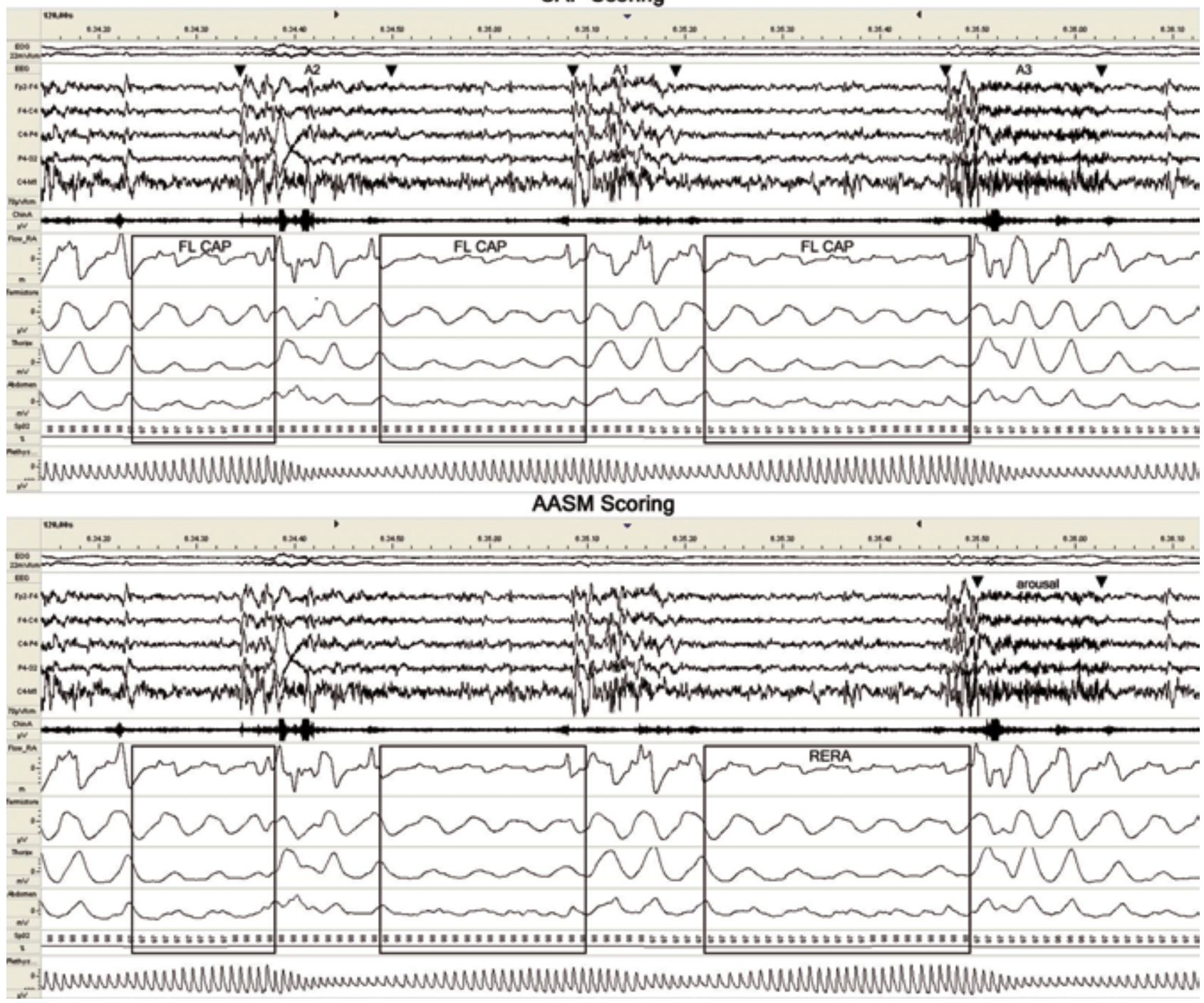

Fig. 2. - Arousal responses to the respiratory events. The flow limitation events are highlighted in the boxes. Flow limitation events associated with the A phase are presented in the top part of the picture (FL CAP boxes). The bottom part of the picture shows, the same three boxes of FL events, but based on the AASM rules only one meets the criteria of RERA. Abbreviations. EOG: electrooculographic channels; EEG: electroencephalographic traces; Chin A: submental electromyogram; Flow RA: nasal air pressure transducer; Termistore: oronasal thermal sensor; Thorax: toracic effort by respiratory inductive plethysmograph; Abdomen: abdominal effort by respiratory inductive plethysmograph; SpO2: oxygen saturation; Plethys: finger photoplethysmogram.

second portion of the event. Hypopnea $(\mathrm{H})$ was defined as a drop by $\geq 30 \%$ in the nasal cannula flow lasting at least 10 seconds and associated with a $\geq 4 \%$ desaturation.

FL event (FL) was defined as a sequence of breaths, not meeting criteria for AP or $\mathrm{H}$, lasting at least 10 seconds, characterized by increasing respiratory effort (assessed by inductance plethysmography) and/or by flattening of the inspiratory portion of the nasal flow trace. Scoring of FL events was manually performed (Figures 2-3).

\section{Scoring of EEG}

Sleep stages were scored according to the AASM rules(Iber $C$ et al. 2007). The definitions of EEG activation and EEG arousal were based respectively on CAP rules (Terzano et al. 2002) and AASM criteria (Iber C et al. 2007). The CAP measures and EEG arousals were visually detected and automatically quantified with the

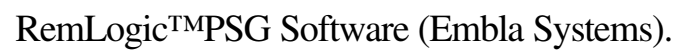

The following CAP variables were measured: CAP time (CAP time in NREM sleep), CAP rate (the per- 


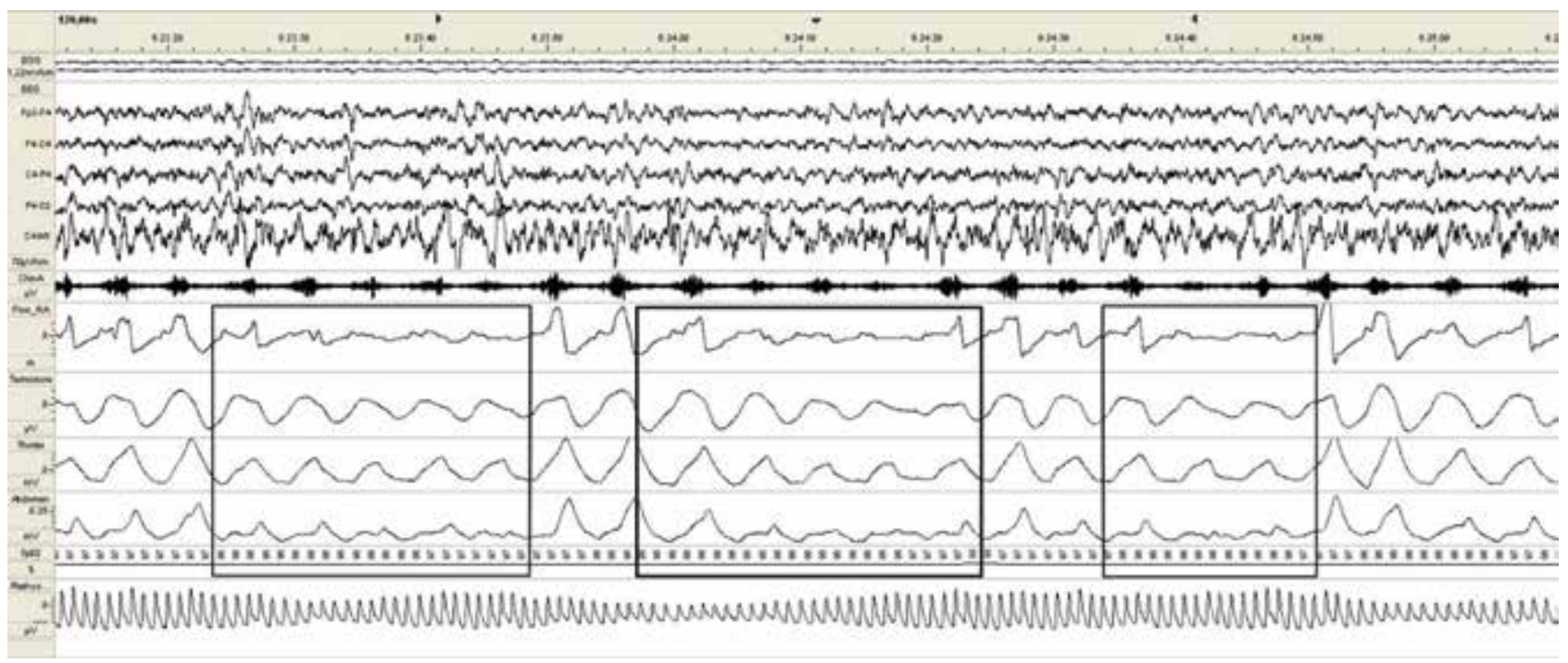

Fig. 3. - Respiratory events without EEG activation. In the boxes are showed non-EEG correlated flow limitation events. Abbreviations. EOG: electrooculographic channels; EEG: electroencephalographic traces; Chin A: submental electromyogram; Flow RA: nasal air pressure transducer; Termistore: oronasal thermal sensor; Thorax: toracic effort by respiratory inductive plethysmograph; Abdomen: abdominal effort by respiratory inductive plethysmograph; SpO2: oxygen saturation; Plethys: finger photoplethysmogram.

centage ratio of CAP time to NREM sleep time) in total NREM sleep, and the percentage of each phase A subtype (A1, A2, A3).

The sleep parameters analyzed in our patients were compared with those of normal age- balanced subjects, selected from the database of the Parma University Sleep Disorders Center.

\section{Temporal relation between arousal responses and $F L$ events}

Analysis of cerebral response was focused in a time range of 4 seconds before and 4 seconds after respiratory recovery to FL events (Thomas 2003).

When a CAP A phase occurred in this time interval we considered the FL as associated with CAP (FLCAP) (Figure 2).

When the EEG activation met the criteria for the definition of AASM EEG arousal, the FL was counted also as RERA (Figure 2).

The FL that were not associated to an EEG activation were classified as non-EEG correlated FL (Figure 3).

\section{Statistical analysis}

Analysis was performed during Total Sleep Time (TST), NREM sleep and rapid eye movement (REM) sleep. Data are presented using descriptive statistics. A Kruskal-Wallis ANOVA compared the three groups (normal subjects; moderate-severe OSAS patients; mild OSAS patients). A MannWhitney test was applied when ANOVA reached significance. The level for statistical significance was established at $\mathrm{p}<0.05$. All statistical analyses were performed using IBM SPSS statistic 19.

\section{Results}

The selected PSG recordings showed high variability of the AHI (AHI range: 5-85), with an extensive distribution covering all severity levels of the OSAS pathology. The mild group was composed of 8 patients, while the moderate-severe group included 11 patients. There were no age differences between two OSAS patients groups $(\mathrm{p}=0.2818)$.

Anthropometric, clinical and respiratory measures of the 19 patients are shown in Table I.

The PSG data of the normal subjects and the two groups of OSAS patients are summarized in Table II. No OSAS patient showed a periodic limb movement disorder.

Compared to normal subjects both OSAS groups showed an increase of WASO (wake after sleep onset) and NREM sleep percentage and a reduction of REM sleep percentage, (Table II). The arousal 


\begin{tabular}{|l|c|c|}
\hline \multicolumn{2}{|l|}{ Table I. - Clinical-anthropometric characteristics and polysomnographic respiratory pattern of two patient groups. } \\
\hline & M group & MS group \\
\hline Age (years) & $57,38(8,88)$ & $51,91(11,68)$ \\
\hline Body Mass Index (kg/m2) & $25,04(3,49)$ & $31,48(7,99)$ \\
\hline Neck circunference (cm) & $38,88(2,10)$ & $44,88(5,33)$ \\
\hline Mallampati (n) & $3,57(0,53)$ & $3,25(0,71)$ \\
\hline Epworth Sleepiness Score (n) & $8,13(4,91)$ & $6,00(2,98)$ \\
\hline Apnea Hypopnea Index (n/h) & $8,33(2,84)$ & $44,02(21,09)$ \\
\hline Respiratory Disturbance Index (n/h) & $15,76(5,34)$ & $47,23(18,82)$ \\
\hline Oxygen Desaturation Index (n/h) & $7,00(3,13)$ & $42,37(21,62)$ \\
\hline Average SaO2 (\%) & $95,45(1,15)$ & $93,33(2,70)$ \\
\hline Time SaO2<90\% (\%) & $0,28(0,33)$ & $13,23(19,27)$ \\
\hline Total Sleep Time Spent in Supine Body Position (\%) & $39,13(26,99)$ & $40,05(28,96)$ \\
\hline Events are reported as mean value (SD) per night. Abbreviations. M group: mild group; MS group: moderate-severe group. \\
\hline
\end{tabular}

index both in NREM and REM sleep was significantly increased in OSAS patients regardless of clinical severity.

The CAP time showed a progressive enhancement from normal subjects $(152,5 \pm 20,76)$ to mild $(180,64 \pm 34,76)$ and moderate-severe $(282,27 \pm 58,02)$ OSAS patients. A significant increase of CAP rate was found only in the moderate-severe group. Both mild and moderatesevere OSAS patients showed the same distribution of the phase A subtypes of CAP with an increase of the $\mathrm{A} 3$ percentages and a reduction of $\mathrm{A} 1$ and $\mathrm{A} 2$ percentages compared to normal subjects.

A total of 5113 respiratory events were detected in NREM sleep: 1587 OA, 525 MA, 169 AC, $1181 \mathrm{H}$, 1651 FL. In REM sleep a total of 820 events were identified: 242 OA, 39 MA, 7 AC, 274 H, 258 FL. The distribution of respiratory events in TST, NREM sleep and REM sleep were differently expressed in the two groups of OSAS patients (Table III).

FL: in the mild OSAS group the FL events represented the most frequent features while the moderate-severe group showed a higher percentage of AP and H (Figure 4). When the two severity groups were compared for TST and NREM sleep, a significant difference was found in the amount of AP and $\mathrm{H}$ but not for FL events. No statistical difference occurred in REM sleep (Figure 4).

FLCAP: in NREM sleep, 1246 FL were associated to A phases, without significant difference between the two severity group (Figure 4). 861 of FLCAP ended with the A3 CAP phase and 385 with A1 and A2 CAP phases.
RERA: FL events scored as RERA were 678 in NREM sleep, and 113 in REM sleep. No significant difference was found between the two OSAS patients group (Figure 4).

Non-EEG correlated FL: non-EEG correlated FL were 406 in NREM and 145 in REM sleep. No statistical differences were found between the two groups. The comparison among FL, FLCAP and RERA is also shown in Figure 4. In both OSAS severity groups, FL events were significantly higher than RERA in TST but not in REM sleep. In NREM sleep, FL events and FLCAP were significantly higher than RERA only for mild OSAS.

\section{Discussion}

Compared to normal subjects, both OSAS groups showed a significant fragmentation of sleep (high amount of WASO) and alteration of sleep macrostructure (decrement in REM sleep and an increase in NREM sleep).

While the arousal index was statistically similar in the two OSAS groups, sleep instability, expressed by CAP time, showed a progressive enhancement from normal subjects to mild and moderate-severe OSAS patients. The moderate severe group showed a significant increase of CAP rate and A3 phases, while a normal CAP rate coexisted with a higher amount of A3 subtypes in the mild group.

These results suggest that, under the increasing thrust of breathing impairment (from mild to mod- 


\begin{tabular}{|c|c|c|c|c|}
\hline \multicolumn{5}{|c|}{ Table II. - Comparison between normative subjects and OSAS patients for the sleep parameters. } \\
\hline & $\mathrm{N}$ & M group & MS group & P-value \\
\hline TST (min) & $457,1(24,75)$ & $417,56(46,33)$ & $445,35(77.20)$ & Ns \\
\hline SE (\%) & $88,74(7,14)$ & $80,15(14,92)$ & $84,71(11,60)$ & Ns \\
\hline WASO (min) & $37,79(22,64)$ & $86,63(61,18)$ & $68,55(47,76)$ & $\begin{array}{l}p 0.0180 \\
N<M=M S\end{array}$ \\
\hline SL (min) & $10,50(15,98)$ & $6,75(4,31)$ & $10,50(15,98)$ & Ns \\
\hline NREM\% & $75,64(2,98)$ & $83,91(17)$ & $87.17(7.98)$ & $\begin{array}{l}\mathrm{p} 0.0001 \\
\mathrm{~N}<\mathrm{M}=\mathrm{MS}\end{array}$ \\
\hline REM (\%) & $25,19(2,93)$ & $16,09(8,09)$ & $12,83(9,98)$ & $\begin{array}{l}p<0.0001 \\
N>M=M S\end{array}$ \\
\hline Al in $\operatorname{NREM}(n / h)$ & $16,44(8.83)$ & $32,76(12.95)$ & $44,66(17.49)$ & $\begin{array}{l}\mathrm{p}<0.001 \\
\mathrm{~N}<\mathrm{M}=\mathrm{MS}\end{array}$ \\
\hline Al in REM $(n / h)$ & $16.95(8.30)$ & $37.38(26.67)$ & $38.18(26.82)$ & $\begin{array}{l}\mathrm{P}<0.0382 \\
\mathrm{~N}<\mathrm{M}=\mathrm{MS}\end{array}$ \\
\hline CAP time (min) & $152,5(20,76)$ & $180,64(34,76)$ & $282,27(58,02)$ & $\begin{array}{l}p<0.0001 \\
N<M<M S\end{array}$ \\
\hline CAP rate (\%) & $45,65(7,71)$ & $52,20(10,32)$ & $73,95(14,36)$ & $\begin{array}{l}p<0.0001 \\
N=M<M S\end{array}$ \\
\hline $\mathrm{Al}(\%)$ & $57,11(7,34)$ & $32,95(15,32)$ & $28,76(15,32)$ & $\begin{array}{l}P<0.0001 \\
N>M=M S\end{array}$ \\
\hline A2 (\%) & $29,16(4,30)$ & $11,33(3,00)$ & $13,53(5,329$ & $\begin{array}{l}p<0.0001 \\
N>M=M S\end{array}$ \\
\hline A3 (\%) & $13,73(3,05)$ & $55,71(4,07)$ & $57,71(17,01)$ & $\begin{array}{l}p<0.0001 \\
N<M=M S\end{array}$ \\
\hline \multicolumn{5}{|c|}{$\begin{array}{l}\text { Events are reported as mean value (SD) per night. } \\
\text { Abbreviations. TST: total sleep time; SE: Sleep Efficiency; WASO: wake time after sleep onset; SL: Sleep Latency; NREM\%: per- } \\
\text { centage of time spent in non rapid eye movements sleep; REM\% sleep: percentage of time spent in rapid eye movements } \\
\text { sleep; Al in NREM: number of AASM arousal/ hour of NREM sleep; Al in REM: number of AASM arousal/ hours of REM sleep; } \\
\text { CAP time: total minutes of cycling alternating pattern; CAP rate(\%): Cycling alternating pattern rate; Al (\%):percentage of } \\
\text { CAP-phase A1; A2(\%):percentage of CAP-phase A2; A3(\%):percentage of CAP-phase A3; N: normal subjects; MS group: } \\
\text { moderate-severe group; M group: mild group. } \\
\text { P-values are from non-parametric test (Mann-Whitney): comparisons between the three groups (normative subjects(N), } \\
\text { mild OSAS (M) and moderate-severe OSAS (MS) patients) were made when ANOVA (Kruskal-Wallis) reached significance } \\
\text { (p value < } 0.05 \text { ). }\end{array}$} \\
\hline
\end{tabular}

erate severe OSAS), the sleeping brain exploits the available arousal responses (Younes 2004), shifting from the lower instability and the weaker CAP A1 phases towards the high instability and the more powerful A3 subtypes to interrupt the respiratory events and recover effective breathing.

These data are in agreement with the evidence that the sleeping brain is a complex system that modulates its internal states according to a continuous elaboration of the ongoing inputs. The CAP variables and the traditional PSG parameters represent the gradual adaptive solutions not only in the environment-sleep relationship (Terzano et al. 1990), but also in the regulation of internal homeostasis (Parrino et al. 2012). Through this wide range of reactivity, the sleeping brain selects the different responses that put the system in an flexible balance between sleep maintenance and respiratory functionality. Similar to the rising levels of acoustic perturbation (Terzano et al. 1990), the increase of OSAS severity determines progressive sleep instability and structural alterations as reflected by CAP parameters.

The severity of OSAS affected also the features of respiratory events: compared to AP and H, FL events dominated in the mild group where they represented the prevalent respiratory pattern. As OSAS severity increases, these subtle respiratory events evolve into major respiratory events such as AP and $\mathrm{H}$. A similar progression has been described from upper airway resistance syndrome (UARS) to OSAS (Jonczak et al. 2009). Tamisier et al. (Tamisier et al. 2000) showed a prevalent RERA pattern in UARS, an obstructive 
Table III. - Distribution of respiratory events in sleep;

\begin{tabular}{|l|c|c|c|c|c|c|}
\hline & \multicolumn{2}{|c|}{ TST } & \multicolumn{2}{c|}{ NREM sleep } & \multicolumn{2}{c|}{ REM sleep } \\
\hline & M group & MS group & M group & MS group & M group & MS group \\
\hline OA+MA(\%) & 22,38 & 47,06 & 19,76 & 48,66 & 32,57 & 35,51 \\
\hline H (\%) & 14,71 & 28,26 & 13,08 & 26,69 & 21,07 & 39,67 \\
\hline FL (\%) & 62,91 & 24,68 & 67,16 & 24,65 & 46,36 & 24,82 \\
\hline FL CAP (\%) & & & 50,84 & 18,56 & & \\
\hline RERA(\%) & 31,38 & 8,71 & 33,24 & 8,66 & 24,14 & 9,06 \\
\hline
\end{tabular}

Events are reported as percentage of total respiratory events (AP, $\mathrm{H}$ and $\mathrm{FL}$ )

Abbreviations: TST: total Sleep Time; NREM sleep: non rapid eye movements sleep; REM sleep: rapid eye movements sleep;

OA: obstructive apnea; AM: mixed apnea; H: hypopnea; FL: flow limitation; FL. CAP: flow limitation linked to CAP A -phases;

RERA: respiratory effort related-arousal; M group: mild group; MS group: moderate-severe group.

hypopnea-apnea pattern in severe OSAS and a mixed pattern in moderate OSAS. As an integrative contribution, our data found a prevalent FL pattern in mild OSAS suggesting a possible underestimation of this features in non severe OSAS.

According to number of studies the arousal scoring in OSAS patients is crucial for the analysis of FL events (Thomas 2003; Masa et al. 2013; Jordan et al. 2011; Chandra et al. 2013). However at the moment a shared interpretation of EEG responses to the FL events is lacking. In our patients, only $41.1 \%$ of FL events analyzed in NREM sleep met the AASM criteria for the RERA definition in contrast $75.5 \%$ of FL events presented a CAP A phase at the end of the respiratory event.

These data suggest that the RERA scoring underestimates the disruptive influences of OSAS on sleep and the definition of arousal with the CAP rules provides more information than AASM criteria for the evaluation of cerebral impact of FL events in NREM sleep.

As previously demonstrated for AP and $\mathrm{H}$ (Terzano et al. 1996), our data showed that most FLCAP (69.1\%) terminated with a CAP phase A3 subtype. Nevertheless, also the slow components of CAP (A1-A2 CAP A phases) were well represented (30.9\% FL), confirming spectral EEG analysis studies showing an increase of theta-delta power at the end of AP and $\mathrm{H}$ events in the absence of AASM arousal (Svanborg \& Guilleminault 1996; Black et al. 2000; Dingli et al. 2002).

In TST $28.8 \%$ of FL were not associated with EEG change $(24.5 \%$ in NREM and in $56.2 \%$ in REM), and the majority (51.4\% of the non EEG correlated FL) showed signs of autonomic arousal (Pulse Wave Amplitude drops and increase of heart rate). As described in the literature, arousals which are defined exclusively by autonomic changes, such as a transient increase in blood pressure or heart rate, may have a significant impact on sleepiness in OSAS patients (Martin et al. 1997). In the present study, the daytime consequences of these events without EEG response were not evaluated, as most of our patients did not complain of excessive sleepiness (Table I) and the heart rate response was not measured in detail.

Although the high number of respiratory events allowed us to collect an extensive sample of data, the limitation of the study is a small group of OSAS patients

In the future it will be necessary to collect a more larger number of patients, to clarify the biological price of FL events during sleep in OSAS.

\section{Conclusion}

There are different phenotypes of OSAS (Eckert et al. 2013) which may vary over time, aging, weight shifts, and other events including sleep deprivation, changes in autonomic activation, exposure to intermittent hypoxia. This study aims to add new information about the phenotypes of OSAS, including FL events in the spectrum of obstructive events (simple snoring, RERA, H, AP) (Pépin et al. 2012).

In the estimation of cerebral impact of FL events in NREM sleep, the CAP metrics offer more extensive information than AASM rules. Indeed, although sleep macrostructure and arousal index allow to discriminate between control subjects and OSAS patients, only the CAP measures differentiate the severity of OSAS. 
TST

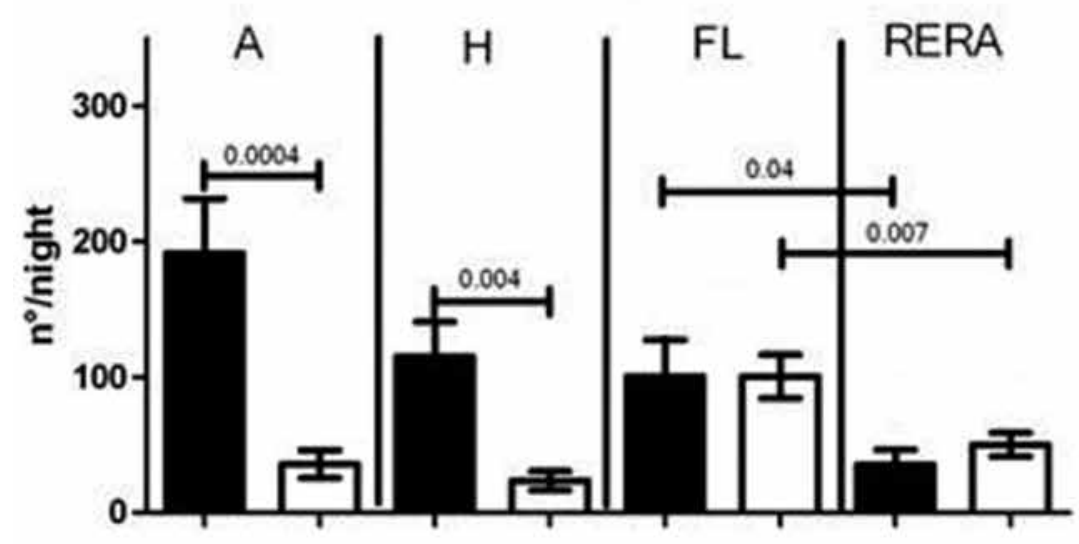

NREM

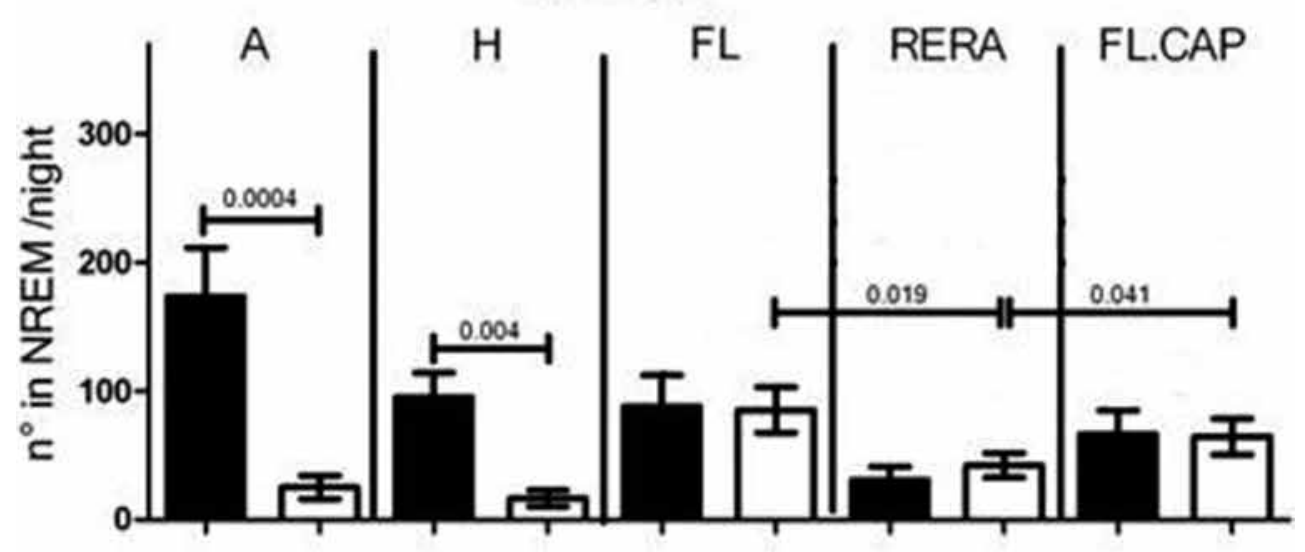

REM

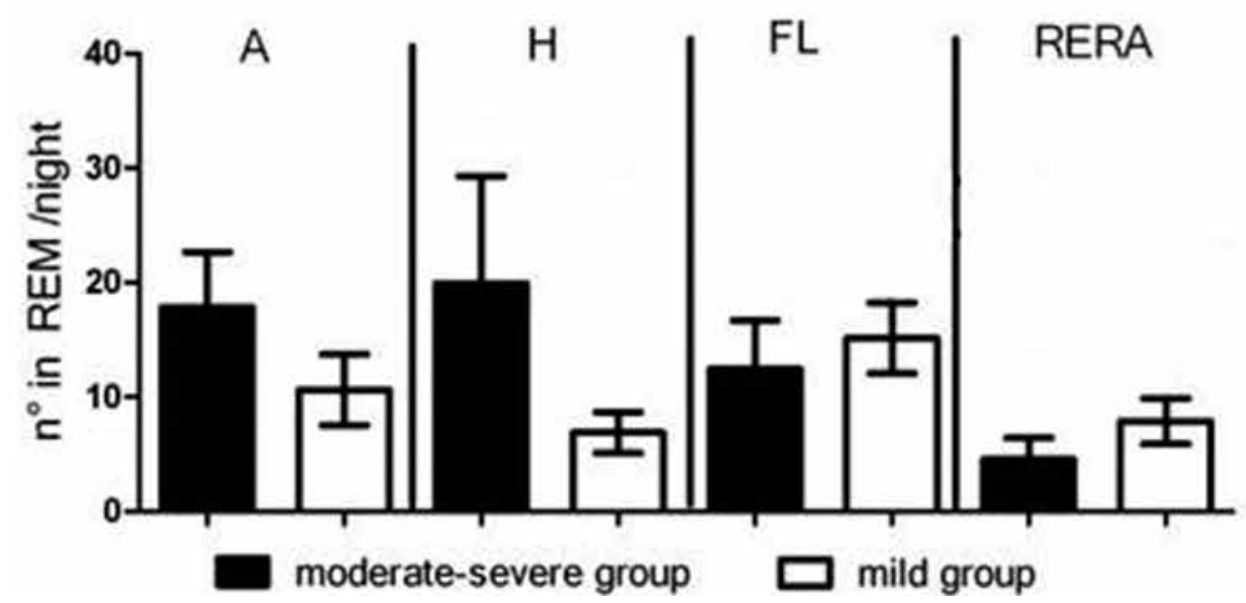

Fig. 4. - Distribution of respiratory events in the two groups of patients in total sleep time, NREM and REM sleep. Events are reported as mean value \pm standard error of the mean (SEM) per night. Statistical analysis by non-parametric test (Mann-Whitney) between the 2 group are reported. Abbreviations TST: total sleep time; NREM sleep: non rapid eye movements sleep; NREM sleep: non rapid eye movements sleep; $A$ : obstructive apnea plus mixed apnea; H: hypopnea; FL: total flow limitation; RERA: flow limitation event linked to AASM arousal; FL CAP: flow limitation linked to CAP A -phases. 
In the future it will be interesting to analyze the relationship between CAP and autonomic responses to respiratory events to understand whether the analysis of autonomic arousal is a reliable surrogate of the CAP scoring. This approach could determine a sensible reduction of gap between PSG and portable monitoring (PM) interpretation.

\section{Disclosure Statement}

This was not an industry supported study. The authors have indicated no financial conflict of interest.

\section{References}

Black J.E., Guilleminault C., Colrain I.M., Carrillo O. Upper airway resistance syndrome. Central electroencephalographic power and changes in breathing effort. Am. J. Respir. Crit. Care Med., 162: 406-411, 2000.

Chandra S., Sica A.L., Wang J., Lakticova V., Greenberg H.E. Respiratory effort-related arousals contribute to sympathetic modulation of heart rate variability. Sleep Breath., 17: 1193-1200, 2013.

Dingli K., Assimakopoulos T., Fietze I., WittC., Wraith P.K., Douglas, N.J. Electroencephalographic spectral analysis: detection of cortical activity changes in sleep apnoea patients. Eur. Respir. J., 20: 12461253, 2002.

Eckert D.J., White D.P., Jordan A.S., Malhotra A., Wellman A. Defining Phenotypic Causes of Obstructive Sleep Apnea: Identification of Novel Therapeutic Targets. Am. J. Respir. Crit. Care Med., 188: 996-1004, 2013.

Iber C., Ancoli-Israel S., Chesson A., Quan S.F. for the American Academy of Sleep Medicine. The AASM manual for the scoring of sleep and associated events: rules, terminology and technical specifications. 2007, Westchester: American Academy of Sleep Medicine.

Jonczak L., Plywaczewski R., Sliwinski P., Bednarek M., Gorecka D., Zielinski J. Evolution of upper airway resistance syndrome. J. Sleep Res., 18: 337-341, 2009.

Jordan A.S., Eckert D.J., Wellman A., Trinder J.A., Malhotra A., White D.P. Termination of respiratory events with and without cortical arousal in obstructive sleep apnea. Am. J. Respir. Crit. Care Med., 184: 1183-1191, 2011.

Martin S.E., Wraith P.K., Deary I.J., Douglas N.J. The effect of nonvisible sleep fragmentation on daytime function. Am. J. Respir. Crit. Care Med., 155: 1596-1601, 1997.
Masa J.F., Corral J., Gomez de Terreros J., DuranCantolla J., Cabello M., Hernandez-Blasco L., Monasterio C., Alondo A., Chiner E., Aizpuru F., Zamorano J., Cano R., Montserrat J.M.; Collaborating group: Garcia-Ledesma E., Pereira R., Cancelo L., Martinez A., Sacristan L., Salord N., Carrera M., Sancho-Chust J.N., Embid C. Significance of including a surrogate arousal for sleep apnea-hypopnea syndrome diagnosis by respiratory polygraphy. Sleep, 36: 249-257, 2013.

Parrino L., Ferri R., Bruni O., Terzano M.G. Cyclic alternating pattern, (CAP): the marker of sleep instability. Sleep Med. Rev., 16: 27-45, 2012.

Pépin J.L., Guillot M., Tamisier R., Lévy P. The upper airway resistance syndrome. Respiration, 83: 559-566, 2012.

Sleep-related breathing disorders in adults: recommendations for syndrome definition and measurement techniques in clinical research. The Report of an American Academy of Sleep Medicine Task Force. Sleep, 22: 667-689, 1999.

Svanborg E., Guilleminault C. EEG frequency changes during sleep apneas. Sleep, 19: 248-254, 1996.

Tamisier R., Pepin J.L., Wuyman B., Smith R.m, Argod J., Levy P. Characterization of pharyngeal resistance during sleep in a spectrum of sleep-disordered breathing. J. Appl. Physiol., 89: 120-130, 2000.

Terzano M.G., Parrino L., Smerieri A., Chervin R., Chokroverty S., Guilleminault C., Hirshkowitz M., Mahowald M., Moldofsky H., Rosa A., Thomas R., Walters A. Atlas, rules, and recording techniques for the scoring of cyclic alternating pattern, (CAP) in human sleep. Sleep Med., 3: 187-199, 2002.

Terzano M.G., Mancia D., Salati M.R., Costani G., Decembrino A., Parrino L. The cyclic alternating pattern as a physiologic component of normal NREM sleep. Sleep, 8: 137-145, 1985.

Terzano M.G., Parrino L., Boselli M., Spaggiari M.C. \& Di Giovanni G. Polysomnographic analysis of arousal responses in obstructive sleep apnea syndrome by means of the cyclic alternating pattern. J. Clin. Neurophysiol., 13: 145-155, 1996.

Terzano M.G., Parrino L., Fioriti G., Orofiamma B. \& Depoortere H. Modifications of sleep structure induced by increasing levels of acoustic perturbation in normal subjects. Electroencephalogr. Clin. Neurophysiol., 76: 29-38, 1990.

Thomas R.J. Arousals in sleep-disordered breathing: patterns and implications. Sleep, 26: 1042-1047, 2003.

Younes M. Role of arousals in the pathogenesis of obstructive sleep apnea. Am. J. Respir. Crit. Care Med., 169: 623-633, 2004. 